\title{
Pseudorabies Virus and Herpes Simplex Virus Type 1 Utilize Different Tegument-Glycoprotein Interactions to Mediate the Process of Envelopment
}

\author{
Omar S. Omar $^{\mathrm{a}}$ Alicia J. Simmons ${ }^{\mathrm{a}}$ Nicole M. Andre ${ }^{\mathrm{a}}$ Duncan W. Wilson ${ }^{\mathrm{b}}$ \\ Sarah T. Gross ${ }^{\text {a }}$ \\ ${ }^{a}$ Department of Biology, Farmingdale State College, Farmingdale, N.Y., and b Department of Developmental and \\ Molecular Biology, Albert Einstein College of Medicine, New York, N.Y., USA
}

\section{Key Words}

PRV $\cdot$ HSV-1 $\cdot$ gH $\cdot$ VP16 $\cdot$ Envelopment $\cdot$ Glycoprotein • Tegument

\begin{abstract}
Background and Objective: During herpesvirus envelopment capsids, tegument polypeptides and membrane proteins assemble at the site of budding, and a cellular lipid bilayer becomes refashioned into a spherical envelope. A web of interactions between tegument proteins and the cytoplasmic tails of viral glycoproteins play a critical role in this process. We have previously demonstrated that for herpes simplex virus (HSV)-1 the cytoplasmic tail of glycoprotein $\mathrm{H}$ $(\mathrm{gH})$ binds the tegument protein VP16. The HSV and pseudorabies virus (PRV) genomes are essentially collinear, and individual gene products show significant sequence homology. However, the demarcation of function often differs between PRV and HSV proteins. The goal of this study was to determine whether PRV $\mathrm{gH}$ and VP16 interact in a manner similar to their homologs in HSV. Methods: A fusion protein pull-down assay was performed in which a PRV gH cytoplasmic tail-glutathione S-transferase fusion protein, bound to glutathione-Sepharose beads, was incubated with PRV-infected cell cytosol, washed and subjected to Western blot analysis using anti-PRV VP16 antisera. Results: Western blots
\end{abstract}

indicate that PRV VP16 does not specifically bind to the PRV $\mathrm{gH}$ tail. Conclusion: Our results highlight that, despite the relatively close evolutionary relationship between HSV and PRV, there are significant differences in their protein interactions that drive envelopment. Copyright $\odot 2012$ S. Karger AG, Basel

The herpesvirus family is composed of over a hundred viruses that infect a broad range of vertebrates. The subfamily Alphaherpesviridae include herpes simplex viruses (HSV-1 and -2), the causative agent of oral and genital legions in humans, and pseudorabies virus (PRV), which is often used as a model system to study alphaherpesvirus infections. The natural host of PRV is the swine, but PRV can infect a broad range of nonprimate mammals.

The herpesvirus virion is composed of a proteinaceous, icosahedral capsid that is assembled and packaged with the double-stranded DNA genome in the nucleus of infected cells. The mature capsid is enveloped within a lipid bilayer derived from host cell membranes, and contains multiple virally encoded glycoproteins that function in many aspects of the viral lifecycle, including host membrane attachment and fusion, immune evasion, and prevention of apoptosis [1]. Between the capsid and the envelope is an amorphous, proteinaceous layer

\section{KARGER}

Fax +4161306 1234

E-Mail karger@karger.ch

www.karger.com
(C) 2012 S. Karger AG, Basel

0300-5526/13/0561-0050\$38.00/0

Accessible online at:

www.karger.com/int
Sarah T. Gross

Department of Biology, Farmingdale State College

2350 Broadhollow Road

Farmingdale, NY 11735 (USA)

Tel. +1 631420 2102, E-Mail sarah.gross@farmingdale.edu 


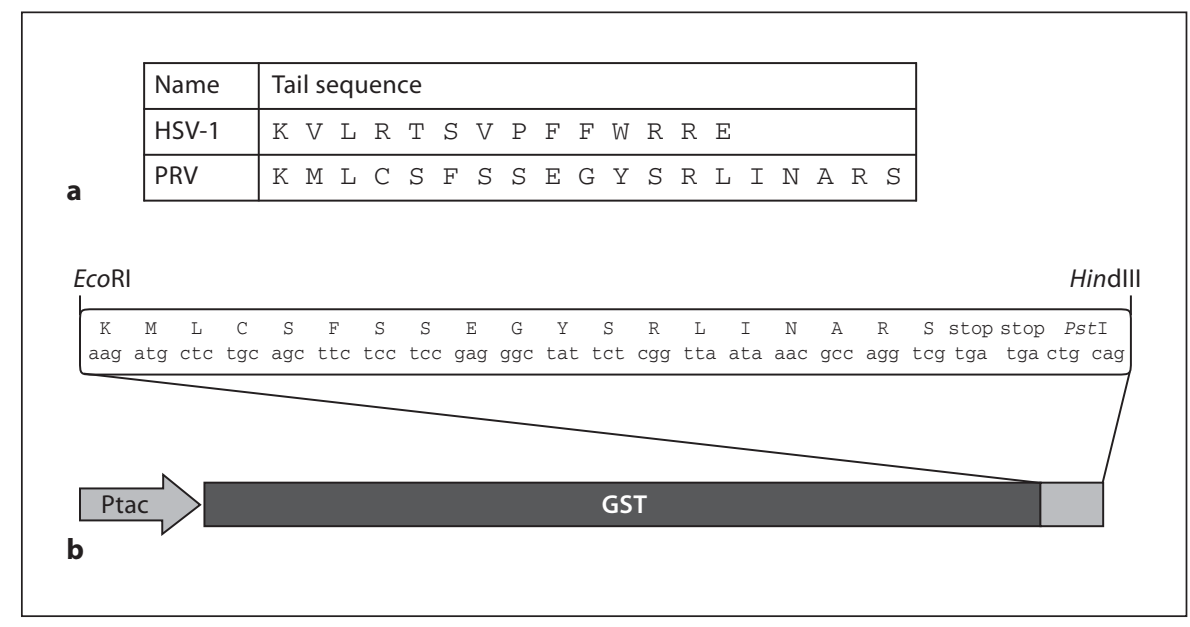

Fig. 1. a Amino acid sequence comparison of the gH cytoplasmic tails of HSV-1 and PRV-1. b Construction of a GST-PRV gH tail fusion protein. A schematic of the fusion protein expression region of plasmid pGEX-KG is shown. Indicated in the lower bar is the IPTG inducible promoter Ptac, the direction of transcription (gray arrow), the GST open reading frame (dark grey), and the polylinker region (light gray bar), flanked by the EcoRI and Hin-

known as the tegument, which is made up of at least 15 different viral proteins, although the functions of many of the tegument proteins remain elusive [1].

Several studies have indicated that viral envelopment and tegumentation occurs at late Golgi or post-Golgi compartments such as TGN and endosomes [2-4]. However, the molecular details that drive envelopment are still poorly understood. It appears that there is a redundant web of interactions between capsid proteins, tegument and glycoproteins. For example, in HSV, the tegument protein VP16 is known to interact with other tegument proteins such as VP22 [5] and vhs [6,7], as well as glycoprotein $\mathrm{H}(\mathrm{gH})[8,9]$ and possibly $\mathrm{gD}$ and $\mathrm{gB}[10]$. Additionally, the HSV tegument proteins UL11 and VP22 have been shown to interact with gE [11]. Also, in PRV, the tegument protein VP22 binds to both gM and gE [12] and simultaneous deletion of both glycoproteins is required to abrogate envelopment and prevent incorporation of VP22 into the mature viral particles [13]. In contrast, for HSV, simultaneous deletion of $\mathrm{gE}$, gI, and $\mathrm{gD}$ are required in order to appreciably affect viral egress and inhibit viral maturation and envelopment [14] suggesting that the importance of various sets of protein-protein interactions differs between the two viruses.

We have previously demonstrated that in HSV, the tegument protein VP16 (product of the UL48 gene) interacts with the cytoplasmic tail of $\mathrm{gH}$ both in vitro and in
dIII restriction sites used in this study. DNA encoding the PRV $\mathrm{gH}$ cytoplasmic tail and the amino acid sequence of the tail are shown above the vector backbone. The tail is fused in frame with GST at the EcoRI site and terminates with two in frame stop codons, followed by the addition of a PstI site for identification purposes prior to the HindIII site, as indicated. Sequences required for bacterial growth and antibiotic selection are not shown. vivo $[8,9]$. In these experiments, HSV VP16 could be specifically recovered from infected cell extracts using a glutathione S-transferase (GST)-gH tail fusion protein. Additionally, in cells transfected with a construct expressing GFP fused to the gH tail, and infected with wild-type HSV, anti-GFP antibodies could be used to coimmunoprecipitate VP16. These experiments also revealed that the interaction between VP16 and the gH carboxy-terminus is temperature-dependent, in that binding only took place at physiological temperature, but not at lower temperatures $[8,9]$.

The aim of this project was to determine whether PRV $\mathrm{gH}$ interacts with VP16 in a manner analogous to HSV $\mathrm{gH}$. This experiment was prompted by the observation that the sequences of the gH tails of HSV and PRV are significantly different from one another (fig. 1a). We wanted to ascertain whether, despite the low sequence homology, there would nonetheless be a functional similarity between the $\mathrm{gH}$ tails of PRV and HSV.

In order to determine whether PRV VP16 and gH interact, a plasmid was generated expressing GST fused to the predicted cytoplasmic tail of PRV gH (fig. 1b) using the GST vector pGEX-KG [15]. The complementary oligonucleotides 5'-AATTCAAGATGCTCTGCAGCTTCTCCTCCGAGGGCTATTCTCGGTTAATAAACGCCAGGTCGTGATGACTGCAGA-3' and 5'-AGCTTCTGCAGTCATCACGACCAGGCGTTTATTAACCG 


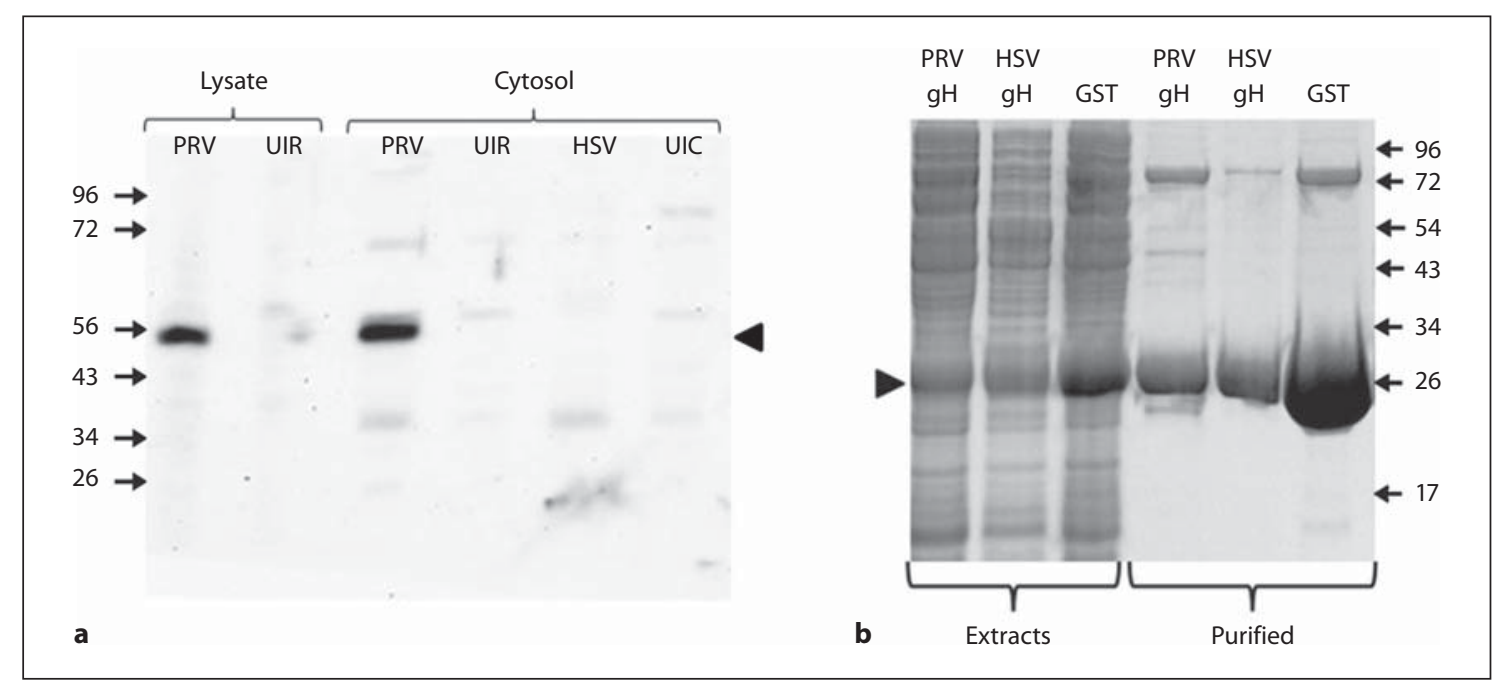

Fig. 2. a Assessing the specificity of the rabbit polyclonal anti-PRV VP16 antisera. Cell lysates and cell cytosol from PRV-infected (PRV) and -uninfected (UIR) RK13 cells, as well as cell cytosol from HSV-infected (HSV) or -uninfected (UIC) COS cells were prepared and analyzed by $10 \%$ SDS-PAGE, followed by Western blotting with the anti-PRV VP16 polyclonal rabbit antiserum at a 1:1,000 dilution. Positions and sizes (in $\mathrm{kDa}$ ) of standard molecular weight markers are indicated at the left of the figure. Arrowhead at the right indicates the position of PRV VP16. b Expression of the GST-PRV gH tail fusion protein. E. coli were transformed

AGAATAGCCCTCGGAGGAGAAGCTGCAGAGCATCTTG-3' were synthesized and annealed, which generates a dsDNA fragment with EcoRI and HindIII ends, and which encodes the PRV gH cytoplasmic tail followed by two stop codons and a unique PstI restriction site for identification purpose. Ligation of this fragment with HindIII/EcoRI digested pGEX-KG DNA results in an in-frame fusion of the GST and $\mathrm{gH}$ tail coding regions at the EcoRI site (fig. 1b).

In order to perform the subsequent PRV VP16-gH tail binding assay described below, it was necessary to generate anti-PRV VP16 polyclonal antisera. Open Biosystems, Inc. synthesized the oligopeptide HLIPRDALNRMFEM corresponding to the $14 \mathrm{C}$-terminal amino acids of PRV VP16, which is rich in hydrophilic amino acids and has low sequence homology to HSV VP16. The purified synthetic peptide was injected into rabbits and the resulting serum was evaluated for its specificity to PRV VP16 (fig. 2a). Cell lysates and cell cytosol from PRV-infected and -uninfected RK13 cells as well as cell cytosol from HSV-infected and -uninfected COS cells (prepared as described below) were resolved by $10 \%$ SDS-polyacrylamide gel electrophoresis (SDS-PAGE), and Western blotted with the parental vector (GST) or with the plasmid encoding a fusion between GST and the $\mathrm{gH}$ tail of either PRV (PRV gH) or HSV (HSV gH) as indicated. Following IPTG induction, total bacterial extracts (Extracts) as well as bacterial extracts that had been incubated for $1 \mathrm{~h}$ at $4^{\circ}$ with glutathione-Sepharose beads (Purified) were subjected to $12 \%$ SDS-PAGE and proteins visualized with Coomassie brilliant blue. Positions and sizes (in $\mathrm{kDa}$ ) of standard molecular weight markers are indicated at the right of the figure. Arrowhead at the left indicates the position of GST and the GST$\mathrm{gH}$ fusions. with the anti-PRV VP16 antisera. Results indicate that the antibody specifically recognizes an approximately $53-\mathrm{kDa}$ protein in the PRV-infected samples, equivalent in size to the predicted molecular weight of PRV VP16. The band was not apparent in uninfected or in HSV-infected samples (fig. 2a), nor was it present in Western blots with preimmune serum (data not shown).

To perform the binding assay, plasmids expressing either GST alone, or GST fusion proteins with either PRV or HSV gH tails were transformed into the Escherichia coli strain BL21 Gold (Stratagene) and bacterial extracts were prepared as previously described [9]. The resulting supernatants were incubated with glutathione-Sepharose beads (GE Healthcare), which had been previously washed twice in PBS. Following a 1-hour incubation at $4^{\circ}$, the beads were washed twice with buffer $1(50 \mathrm{~mm}$ Tris- $\mathrm{HCl}$ $\mathrm{pH} 7.6,150 \mathrm{mM} \mathrm{NaCl}, 0.5 \%$ Triton X-100), then twice with buffer 2 (50 mM Tris-HCl pH 7.6, $1.3 \mathrm{M} \mathrm{NaCl}, 0.5 \%$ Triton $\mathrm{X}-100)$, then twice again with buffer 1 . At this point, both crude bacterial extract as well as bead-bound material were resolved by $12 \%$ SDS-PAGE. In figure 2, Coomassie blue staining (Pierce) shows that fusion proteins of the correct size of approximately $26 \mathrm{kDa}$ were readily appar- 


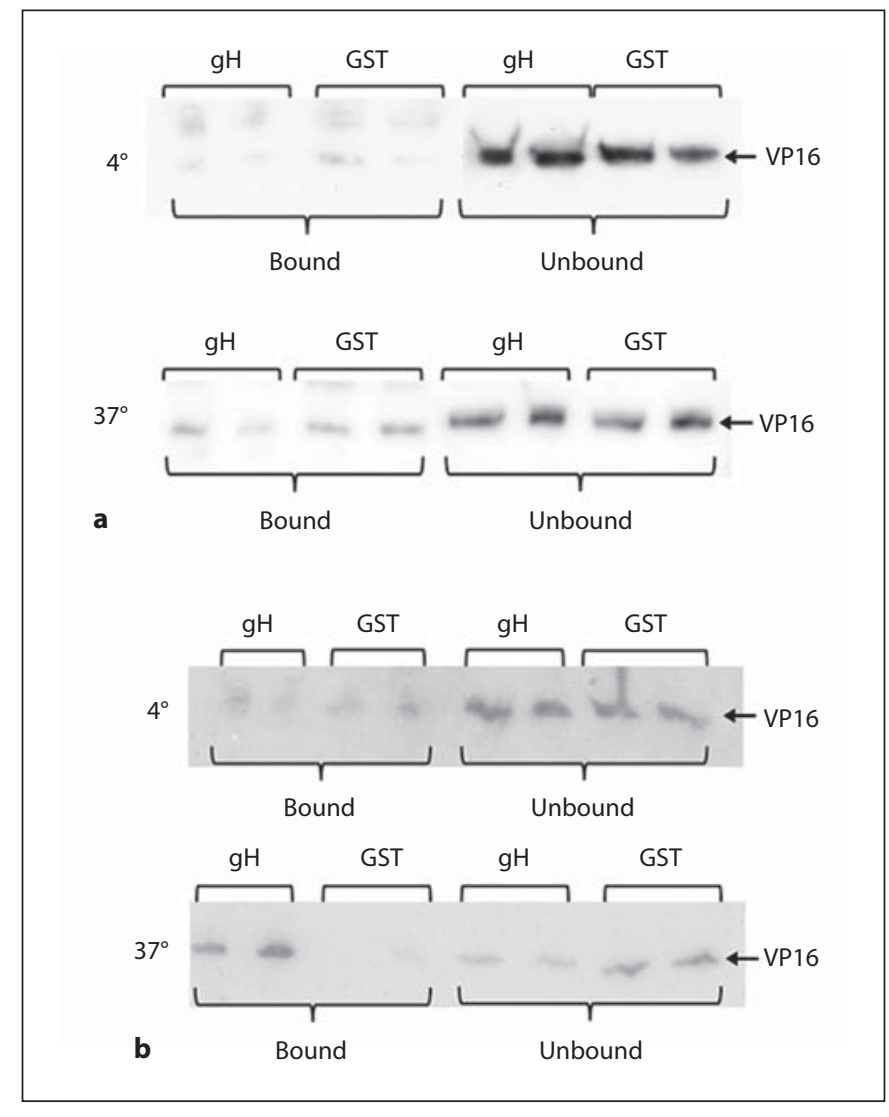

Fig. 3. a Binding of the PRV gH cytoplasmic tail to VP16. GST or GST-PRV gH fusions were induced, bacterial extracts prepared and equivalent amounts of each protein were bound to glutathione-Sepharose beads. These beads were then incubated in duplicate with cytosol prepared from PRV-infected RK13 cells for $1 \mathrm{~h}$ at 4 or $37^{\circ}$, as indicated. Bound or unbound polypeptides were resolved by $10 \%$ SDS-PAGE, transferred to a PVDF membrane, and probed with anti-PRV VP16 antibodies, as indicated. Similar results were obtained with overnight incubations at the respective temperatures. b Binding of the HSV gH cytoplasmic tail to VP16. As a positive control, GST or GST-HSV gH fusions were induced, bacterial extracts prepared and equivalent amounts of each protein were bound to glutathione-Sepharose beads. These beads were then incubated in duplicate with cytosol prepared from $\mathrm{HSV}$-infected COS cells for $1 \mathrm{~h}$ at 4 or $37^{\circ}$, as indicated. Bound or unbound polypeptides were resolved by $10 \%$ SDS-PAGE, transferred to a PVDF membrane, and probed with anti-HSV VP16 antibodies, as indicated.

ent in the bacterial extracts (fig. 2b 'Extracts') and were specifically isolated and purified after incubation of the bacterial extracts with glutathione-Sepharose beads (fig. 2b 'Purified').

The washed beads, bearing specifically-bound GST, GST-PRV gH tail or GST-HSV gH tail fusion proteins were then incubated with cytosol prepared from PRV- infected RK13 cells or HSV-infected COS cells as the source of the tegument protein, VP16. The PRV- and HSV-infected cell cytosols were prepared as follows: confluent monolayers of either RK13 or COS cells were infected at an MOI of 10 with PRV (strain K26 GFP) or HSV-1 (strain SC16), respectively. The infected cells were incubated for $16-18 \mathrm{~h}$ at $37^{\circ}$, and cell cytosol was prepared as previously described [9]. The PRV- and HSVinfected cell cytosols were incubated at various temperatures and times with Sepharose-bound GST fusion proteins. At the end of the incubation period the beads were recovered, washed three times in buffer 1 (see above) and bead-bound and unbound material examined following SDS-PAGE and Western blotting for PRV VP16 or HSV VP16, as previously described [4]. Additionally, binding reactions were resolved by $12 \%$ SDS-PAGE and stained with Coomassie brilliant blue to determine that equivalent levels of the GST fusion proteins were present in this experiment (data not shown).

Western blots indicated that regardless of the temperature or length of incubation, PRV VP16 did not specifically bind to the PRV gH tail/GST fusion protein (fig. 3a). As a positive control, we performed the binding assay with HSV gH/VP16 alongside our PRV binding assay. As expected, we found that the HSV gH tail interacts with HSV VP16 at $37^{\circ}$ but not at $4^{\circ}$ (fig. $3 \mathrm{~b}$ ), consistent with our previous findings $[8,9]$. These results imply that PRV VP16 does not bind to gH under our established conditions.

These experiments were prompted by the observation that the $\mathrm{gH}$ tail of HSV and PRV are significantly different from one another (fig. 1a). Our results highlight that despite the relatively close evolutionary relationship between HSV and PRV, each virus utilizes a different set of tegument-glycoprotein tail interactions to mediate the process of envelopment.

Current studies are focused on identifying tegument binding partners of the cytoplasmic tail of PRV gH. Additionally, new GST fusion constructs are being developed with the cytoplasmic tail of PRV gD as well as other PRV glycoprotein tails to identify other glycoprotein-tegument interactions that may be involved in PRV envelopment.

\section{Acknowledgements}

This work was supported by start-up funds provided by Farmingdale State College, State University of New York (to S.T.G) and by NIH R01 AI083285 (to D.W.W.). We thank Lily Huang and Denise Hopkins for technical assistance. 


\section{References}

1 Knipe DM, Howley PM (ed): Field's Virology, ed 4. Philadelphia, Lippincott Williams \& Wilkins, 2001, vol 2, pp 2399-2459.

2 Pasdeloup D, Beilstein F, Roberts AP, McElwee M, McNab D, Rixon FJ: Inner tegument protein pUL37 of herpes simplex virus type 1 is involved in directing capsids to the trans-Golgi network for envelopment. J Gen Virol 2010;91:2145-2151.

-3 Sugimoto K, Uema M, Sagara H, Tanaka M, Sata T, Hashimoto Y, Kawaguchi Y: Simultaneous tracking of capsid, tegument, and envelope protein localization in living cells infected with triply fluorescent herpes simplex virus-1. J Virol 2008;82:5198-5211.

4 Harley CA, Dasgupta A, Wilson DW: Characterization of herpes simplex virus-containing organelles by subcellular fractionation: role for organelle acidification in assembly of infectious particles. J Virol 2001; 75:1236-1251.

5 Elliott G, Mouzakitis G, O’Hare P: VP16 interacts via its activation domain with VP22, a tegument protein of herpes simplex virus, and is relocated to a novel macromolecular assembly in coexpressing cells. J Virol 1995; 69:7932-7941.
6 Schmelter J, Knez J, Smiley JR, Capone JP: Identification and characterization of a small modular domain in the herpes simplex virus host shutoff protein sufficient for interaction with VP16. J Virol 1996;70:2124-2131.

7 Smibert CA, Popova B, Xiao P, Capone JP, Smiley JR: Herpes simplex virus VP16 forms a complex with the virion host shutoff protein vhs. J Virol 1994;68:2339- 2346.

-8 Kamen DE, Gross ST, Girvin ME, Wilson DW: Structural basis for the physiological temperature dependence of the association of VP16 with the cytoplasmic tail of herpes simplex virus glycoprotein H. J Virol 2005; 79:6134-6141.

-9 Gross ST, Harley CA, Wilson DW: The cytoplasmic tail of herpes simplex virus glycoprotein $\mathrm{H}$ binds to the tegument protein VP16 in vitro and in vivo. Virology 2003;317: $1-12$.

10 Zhu Q, Courtney RJ: Chemical cross-linking of virion envelope and tegument proteins of herpes simplex virus type 1 . Virology 1994; 204:590-599.
1 Farnsworth A, Wisner TW, Johnson DC: Cytoplasmic residues of herpes simplex virus glycoprotein gE required for secondary envelopment and binding of tegument proteins VP22 and UL11 to gE and gD. J Virol 2007; 81:319-331.

12 Fuchs W, Klupp BG, Granzow H, Hengartner C, Brack A, Mundt A, Enquist LW, Mettenleiter TC: Physical interaction between envelope glycoproteins $\mathrm{E}$ and $\mathrm{M}$ of pseudorabies virus and the major tegument protein UL49. J Virol 2002;76:8208-8217.

13 Brack AR, Dijkstra JM, Granzow H, Klupp BG, Mettenleiter TC: Inhibition of virion maturation by simultaneous deletion of glycoproteins $\mathrm{E}, \mathrm{I}$, and $\mathrm{M}$ of pseudorabies virus. J Virol 1999;73:5364-5372.

14 Farnsworth A, Goldsmith K, Johnson DC: Herpes simplex virus glycoproteins $\mathrm{gD}$ and $\mathrm{gE} / \mathrm{gI}$ serve essential but redundant functions during acquisition of the virion envelope in the cytoplasm. J Virol 2003;77:84818494.

15 Guan KL, Dixon JE: Eukaryotic proteins expressed in Escherichia coli: an improved thrombin cleavage and purification procedure of fusion proteins with glutathione Stransferase. Anal Biochem 1991;192:262267. 